\title{
DA METHOD AND SYMPLECTIFICATION FOR FIELD MAP GENERATED MATRICES OF SIBERIAN SNAKE IN RHIC
}

\author{
$\underline{\text { M. Xiao }}^{\#}$, T. Katayama, CNS, U. of Tokyo/RIKEN, Japan \\ E. Forest, KEK, Japan
}

\begin{abstract}
The simulation of spin precession and orbital motion in Siberian Snakes in RHIC based on the calculated or measured numerical magnetic field has been done. DA (Differential Algebraic) approach was introduced to calculate the orbital map of full Siberian Snakes to second order in the phase space variables. The symplectification was done for the maps obtained by DA. The symplectified maps that satisfy exactly the symplectic conditions will be presented in this paper.
\end{abstract}

\section{INTRODUCTION}

During the acceleration of polarized proton beam in RHIC, it is necessary to preserve the spin orientation to a high degree. For this reason, we must include spin tracking and the effect of the proposed Siberian snakes in simulations. Spin precession of the proton depends on the ambient magnetic field and thus it is coupled with the orbital motion. The spin matrix, a rotation in spin space, provides the required rotation of the spin and the correct angle of the precession axis. The Siberian Snake was designed to turn the direction of spin by $180^{\circ}$ with minimal effect on orbital motion.

The laws of physics require that the spin matrix be unitary and the orbit matrix be symplectic. In fact, the entire non-linear orbit map must be symplectic. In general it is not clear that an approximate solution for the spin matrix and the orbital map will produce unitary matrices for the spin and symplectic maps for the orbital part. The violation of the (unitary/symplectic) condition becomes unacceptable whenever the simulation code is used in an iterative mode, i.e., whenever several turns of a machine are tracked. A violation of the condition then leads to a growth or shrinkage of the phase space; occasionally it leads to more complex but still unphysical behavior. It is this issue that motivates the present paper.

The issue of symplectic integration has been addressed by several authors [1][2]. Grossly speaking, symplectic integrators can be divided into the explicit and implicit types. Most accelerator physics codes, of the "kick-code" variety, are explicit integrators. These codes are fast and

"Work supported by RHIC-Spin Project in RIKEN.

"Email: xiao@ rikaxp.riken.go.jp conceptually simple because a variety of effects can be added to them in a more or less self-consistent way. Unfortunately, it seems very hard to produce explicit symplectic integrators for a non-ideal magnetic field, the complexity is compounded by the absence of an analytical representation for the magnetic field.

Therefore, we have opted here to keep using an ordinary integrator, from which we extract a map. This has a small advantage: because the integrator is not symplectic, any violation of the symplectic condition will be a reflection of the integration accuracy or of the non-Maxwellian aspects of the fields. In other words it gives us an idea of the absolute error before any symplectic "fudging" algorithm masks it.

The DA (Differential Algebra) approach [3][4] pioneered by Berz in beam physics permits the calculation of a truncated Taylor map of an arbitrary element to any order, and the Taylor coefficients of the resulting truncated map will be accurate to machine precision. However, since the magnetic field given numerically and interpolated by spline function is smooth in the first derivatives and continuous in the second derivatives, it is possible to introduce DA methods to calculate the map of the full snake to the second order in the phase space variables without refitting the fields.

In fact, the matrices calculated from a field map are not symplectic in general. This is a problem resulting not only from the method we used, but also from the deviation of the magnetic field from the Maxwellian property. The symplectification for the maps obtained by the DA approach will be introduced and symplectified maps that satisfy exactly the symplectic conditions will be presented in this paper.

\section{6-D MAP OF ORBITAL MOTION BY DA APPROACH}

The DA(Differential Algebra) approach is a technique to propagate the derivatives of a function $f\left(x_{i}\right)$ systematically through mathematical transformations on $f$ by simply applying the familiar sum, product and chain rules of differentiation[4]. The derivatives of any complicated function, which may be obtained by successive mapping, can be calculated by extending any function $f$ to a vector $f$ called "DA-vectors". For maptracking, all phase-space coordinates $z_{i}$ become the DA 
vectors $z_{i}$. The first element of $z_{i}$ contains the current value of the coordinates $z_{i}$, and the subsequent elements contain the derivatives with respect to the initial values of $z_{i}$. The vectors $z_{i}$ are initialized by setting the first element to the initial value of $z_{\mathrm{I}}$, the element which contains the first derivative with respect to the $i^{\text {th }}$ initial coordinate is set to be unity, and all the other elements are zero. Each mathematical operation that involves phase-space variables is replaced by a vector operation. Then, a truncated Taylor map

$$
\vec{z}_{\text {final }}=\underset{j_{1}+j_{2}+\cdots+j_{n} \leq N}{\vec{a}_{j_{1}, j_{2}}, \cdots, j_{n}} \prod_{i} z_{i}^{j_{i}}
$$

can be obtained. The LBNL version of the DA package written by Berz was introduced, and Program DASSSTRA was written to do the simulation. In order to make use of DA, the magnetic field was reconstructed again to the second order,

$$
\begin{gathered}
B_{w}(x, y, s)=b_{w}(x, y, s)+\frac{\partial b_{w}(x, y, s)}{\partial x} \cdot x \\
+\frac{\partial b_{w}(x, y, s)}{\partial y} \cdot y+\frac{1}{2} \cdot \frac{\partial^{2} b_{w}(x, y, s)}{\partial x^{2}} \cdot x^{2} \\
+\frac{\partial^{2} b_{w}(x, y, s)}{\partial x \partial y} \cdot x y+\frac{1}{2} \cdot \frac{\partial^{2} b_{w}(x, y, s)}{\partial y^{2}} \cdot y^{2}+\cdots \\
w=x, \quad y, \quad s
\end{gathered}
$$

Where $b_{w}(x, y, s)$ as well as its first and second derivatives are expressed by spline interpolation functions [5] fitted from the numerical field data. Since the spline function is smooth in the first derivatives and continuous in the second derivatives, the truncated Taylor map to the second order can be obtained around any ray started from the entrance of the snake.

The magnetic field strength of Siberian Snake was optimized first in order to make the spin precession turn $180^{\circ}$ and the spin matrix of the snake close to unitary as possible for the particle with the energy $E=250 \mathrm{GeV}$ (storage mode)[5]. Then, six-dimensional map with the variables of $\left(x, p_{x}, y, p_{y},-c t, p_{\mathrm{t}}\right)$ [7] to the second order around any ray can be extracted automatically for the particles with the energy from $\mathrm{E}=25 \mathrm{GeV}$ to $\mathrm{E}=250 \mathrm{GeV}$. Linear matrix $M_{T}$ for the motion at $\mathrm{E}=250 \mathrm{GeV}$ around the central ray was listed in Table 1.

\section{SYMPLECTIFICATION}

\subsection{Symplectic condition checking}

A $2 n \times 2 n$ matrix, $M$, is said to be symplectic if $\mathrm{M}^{\mathrm{T}} \cdot \mathrm{J} \cdot \mathrm{M}=\mathrm{J}$, where $\mathrm{M}^{\mathrm{T}}$ is the transpose of $\mathrm{M}$, and $\mathrm{J}$ is the matrix

$$
M_{T}=\left[\begin{array}{ccccc}
0.99987402 & 12.2799388 & 0.00007164 & \\
-0.0002035 & 0.99987606 & -0.00000085 & \\
-0.00002987 & 0.00014282 & 0.99947619 & \ldots \\
-0.00001740 & -0.00013731 & -0.00008627 & \\
0 . & 0.00000009 & 0 . & \\
0 . & 0 . & 0 . & \\
& & -0.00029680 & 0 . & 0.00000018 \\
& -0.00012853 & 0 . & 0 . \\
& 12.2777225 & 0 . & 0.00000012 \\
& 0.99946440 & 0 . & 0.00000003 \\
& -0.00000006 & 1 . & 0.00020931 \\
& 0 . & 0 . & 1 .
\end{array}\right]
$$

Table 1. The matrix $M_{T}$ obtained by DA before symplectified.

$$
J=\left[\begin{array}{cc}
0 & I \\
-I & 0
\end{array}\right]
$$

Here each entry in $\mathrm{J}$ is a $n \times n$ matrix, I denotes the $n \times n$ identity matrix, and all the other entries are zero. It follows that a symplectic matrix has a unit determinant. The check of the symplecticity for maps of the snake was done, and the results show that it does not satisfy the symplectic conditions completely, although a deviation of the determinant of $M_{T}$ from 1 is as small as $2.8199 \times 10^{-14}$. The maximum error among the matrix elements is $-1.2853 \times 10^{-5}$. The Maxwellian property of the magnetic field around the central ray was checked and it was found that the maximum error is around $10^{-2}$, the same order as the symplectic property of the orbital matrix.

\subsection{Symplectification}

The Dragt-Finn factorisation states that a map near the identity can be expressed as follow [1]:

$$
M_{K}=\exp (\vec{F} \cdot \nabla) I_{d}
$$

Where $I_{d}$ is identity map. The Poisson bracket operator, $[f, g]$, is a special case of the general vector field operator

$$
: f: \vec{g}=[f, g]=\nabla f^{T} \cdot J \cdot \nabla \vec{g}=-\underbrace{J \nabla f}_{\vec{F}} \nabla \vec{g},
$$

Where $\vec{g}$ is an arbitrary vector function of phase space. Then,

$$
f=\int_{0}^{x} J \cdot \vec{F} \cdot d x^{\prime}
$$

If $\vec{F}$ is symplectic, then this computation involves an integral of a curl free function. Thus, the function $f$ is 
unique. If $\vec{F}$ is slightly non-symplectic, then the function $f$ is one of possible symplectifications of the vector field $\vec{F}$, and it will depend on the path of the integration.

We know that the map $M$ (including second order) from the entrance to exit of the snake is nearly the map for a drift of the length equal to the full snake. Therefore, one needs to construct a new map $M_{K}$

$$
M_{k}=D^{-1}\left(\frac{L}{2}\right) \cdot M \cdot D^{-1}\left(\frac{L}{2}\right),
$$

Where $D(L / 2)$ is a drift of half-length of the full snake. So the linear part of the $M_{K}$ is a matrix near the identity. In the program DA-SSSTRA, a possible symplectic matrix $M_{K_{\text {ssm }}}$ for $M_{K}$ was computed by an iterative process as follows. First, we compute the vector $\vec{F}$ in such a way that

$$
M_{K}=\exp (\vec{F} \cdot \nabla) I_{d}
$$

Later, we will find a Poisson bracket approximating this general vector field. We then compute

$$
\text { vectorm }=\exp \left(-\vec{F}_{j} \cdot \nabla\right) \cdot M_{K}-I_{d}
$$

as a correction of $\vec{F}$ and check whether vectorm is close enough to zero. If not, then we set

$$
\vec{F}_{\mathrm{j}+1} \Leftarrow \vec{F}_{\mathrm{j}}+\text { vectorm }
$$

and repeat the iteration.

Notice that this computation does not separate the map to be symplectified into the first and second orders, so it can be used for the symplectification of arbitrary order.

Once, :f: is obtained from $\vec{F}$, the resulting symplectified map is

$$
\begin{aligned}
M_{\text {sym }} & =D^{-1}\left(\frac{L}{2}\right) \cdot M_{k_{-} s y m} \cdot D^{-1}\left(\frac{L}{2}\right) \\
& =D^{-1}\left(\frac{L}{2}\right) \cdot \exp (: f:) I_{d} \cdot D^{-1}\left(\frac{L}{2}\right)
\end{aligned}
$$

The maps for the Siberian Snake generated by numerical magnetic field including second order were symplectified, and the symplectified linear matrix was listed in Table 2.

The symplectification results show that there is a little adjustment for every element of the linear map of the snake calculated by DA to be symplectic, but for the second order map, the elements corresponding to the cross terms such as $x_{0} \bullet p_{x o}$ change significantly.

Further work is being done to put the symplectified map into the full lattice of RHIC for spin tracking. The accuracy will have to be gauged in an actual run.

$$
M_{T_{-} s y m}=\left[\begin{array}{cccc}
0.99987402 & 12.27993880 & -0.00000235 \\
-0.0002035 & 0.99987606 & -0.00000912 \\
-0.00004410 & 0.00020831 & 0.99947619 \\
-0.00000913 & -0.00010970 & -0.00008626 \\
0 . & 0.00000013 & -0.00000001 \\
0 . & 0 . & 0 . \\
& -0.00036238 & 0 . & 0.00000013 \\
& -0.00015614 & 0 . & 0 . \\
12.27772254 & 0 . & 0.00000000 \\
& 0.99946440 & 0 . & 0.00000001 \\
& -0.00000018 & 1 . & 0.00020931 \\
& 0 . & 0 . & 1 .
\end{array}\right]
$$

Table 2. The matrix after symplectifed

\section{REFERENCES}

[1] A. J. Dragt, AIP Conference Proceedings, 1987.

[2] E. Forest, et al., KEK Report 92-14, September 1992

[3] M Berz, Particle Accelerators, Vol. 24, 1989.

[4] F.Willeke, Proceedings of the CAS (CERN Accelerator School) Fifth Advanced Accelerator Physics Course, CERN 95-06(1995), Greece 1993.

[5] M. Xiao, et al., Spin note AGS/RHIC/SN No. 078, September 10, 1998.

[6] I.Alekseev,et al, Design Manual , Polarized Proton Collider at RHIC, July, 1998 .

[7] F.C.Iselin,The MAD Program Physical Methods Manual. Geneva, Switzerland, January, 1994. 